\title{
Interlayer Coupling in EuS-Based Superlattices Deduced from Neutron Scattering Experiments
}

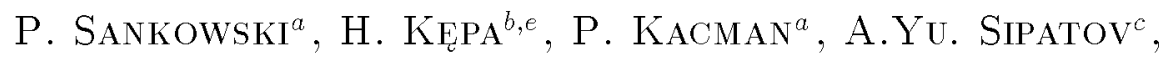 \\ C.F. MajkrzaK ${ }^{d}$ And T.M. Giebultowicz ${ }^{e}$
}

${ }^{a}$ Institute of Physics PAS and ERATO Semiconductor Spintronics Project

al. Lotników 32/46, 02-668 Warsaw, Poland

${ }^{b}$ Institute of Experimental Physics, Warsaw University

Hoża 69, 00-681 Warsaw, Poland

${ }^{c}$ Kharkov Polytechnic Institute, 21 Frunze St., Kharkov, Ukraine

${ }^{d}$ National Institute of Standards and Technology, Gaithersburg, USA

${ }^{e}$ Physics Department, Oregon State University, Corvallis, USA

The ferromagnetic/diamagnetic semiconductor superlattices, EuS/PbS and EuS/YbSe, were studied by neutron reflectivity. In order to determine the strength of the interlayer coupling, the intensity of the first magnetic Bragg peak vs. applied external magnetic field was measured. Additionally, the in-plane anisotropy and the domain structure were studied by polarized neutron reflectivity. The dependence of the intensity of the antiferromagnetic neutron reflectivity peak vs. magnetic field was simulated using a Stoner-Wohlfarth model. To reproduce the observed spectra it was necessary to take into account the presence of fluctuations of the nonmagnetic layers thickness, by assuming a Gaussian spread of the interlayer coupling constant $J$. For both EuS/PbS and EuS/YbSe superlattices, the best fit was obtained for the directions of the in-plane easy axes, which agree with those determined by polarized neutron reflectivity.

PACS numbers: 75.70.Ak, 75.25.+z, 68.65.Cd 


\section{Introduction}

The all-semiconductor superlattice (SL) systems consisting of magnetic EuS layers (bulk EuS is a classical Heisenberg ferromagnet with the Curie temperature of $16.6 \mathrm{~K}$ ) separated by nonmagnetic, and virtually nonconducting (e.g., PbS or $\mathrm{YbSe}$ ) spacer layers exhibit a range of intriguing magnetic properties. In our previous studies $[1,2]$ it was shown by both, the neutron diffraction and neutron reflectivity, that in these structures pronounced interlayer coupling (IC) correlates antiferromagnetically the spins in consecutive ferromagnetic (FM) EuS layers. In some of the EuS/PbS samples the existence of antiferromagnetic (AFM) IC was also confirmed by direct magnetization (SQUID) measurements $[1,3]$. To explain these interlayer spin correlations a model, in which the exchange interactions are mediated by valence band electrons, has been proposed $[4,5]$. The model does not assume any particular interaction mechanism, but attributes IC to the sensitivity of the SL electronic energies to the magnetic order in the magnetic layers, i.e., accounts globally for the spin-dependent band structure effects. In the calculations presented in [4] and [5] the spin-orbit interactions were neglected. Here we improve the model by including the spin-orbit terms in the description of the band structure of $\mathrm{PbS}$, where they are known to play an important role. These corrections do not introduce, however, any significant changes in the obtained previously results.

In order to calculate IC, the total energies of the valence electrons in two cases, one for SL with the same (FM) and the other with opposite (AFM) spin configurations in consecutive magnetic layers, were compared. The obtained sign of $\Delta E$, i.e., of the difference between these two energies per unit surface of the layer, has shown that in both types of SL, EuS/PbS, and EuS/YbSe, the AFM alignment of magnetization vectors in successive magnetic layers is energetically preferred - in agreement with the experimental findings. The IC constant $J$, which describes the strength of the interlayer magnetic coupling resulting from band structure effects, can be obtained directly from the energy difference $\Delta E$. In Ref. [5] it was shown that the strength of the coupling in both studied SL decreases exponentially with the nonmagnetic layer thickness, but for EuS layers separated by $\mathrm{YbSe}$ the calculated $\mathrm{IC}$ is weaker and its range is shorter than for $\mathrm{EuS} / \mathrm{PbS}$ $\mathrm{SL}$. The comparison of the obtained values with the experiment is, however, by far not trivial.

\section{The strength of interlayer coupling}

To determine experimentally the strength of the AFM IC in EuS-based systems, the measurements of the intensity of the first magnetic SL Bragg peak vs. applied external magnetic field were carried out at NIST Center for Neutron Research, at the NG-1 reflectometer using unpolarized neutron beam. The intensity of this peak is directly related to the relative configuration of the magnetizations 
in the consecutive magnetic layers. The magnetic field, applied in the growth plane of the samples, forces the gradual change of the direction of the magnetizations in adjacent EuS layers leading eventually to the FM alignment of the initially antiferromagnetically coupled layers. Due to parallel alignment of all EuS layer magnetizations in the applied field, the AFM peak disappears and the increased intensity of the structural peak is visible (Fig. 1), because the magnetic period of the FM ordered layers and the SL period are equal.

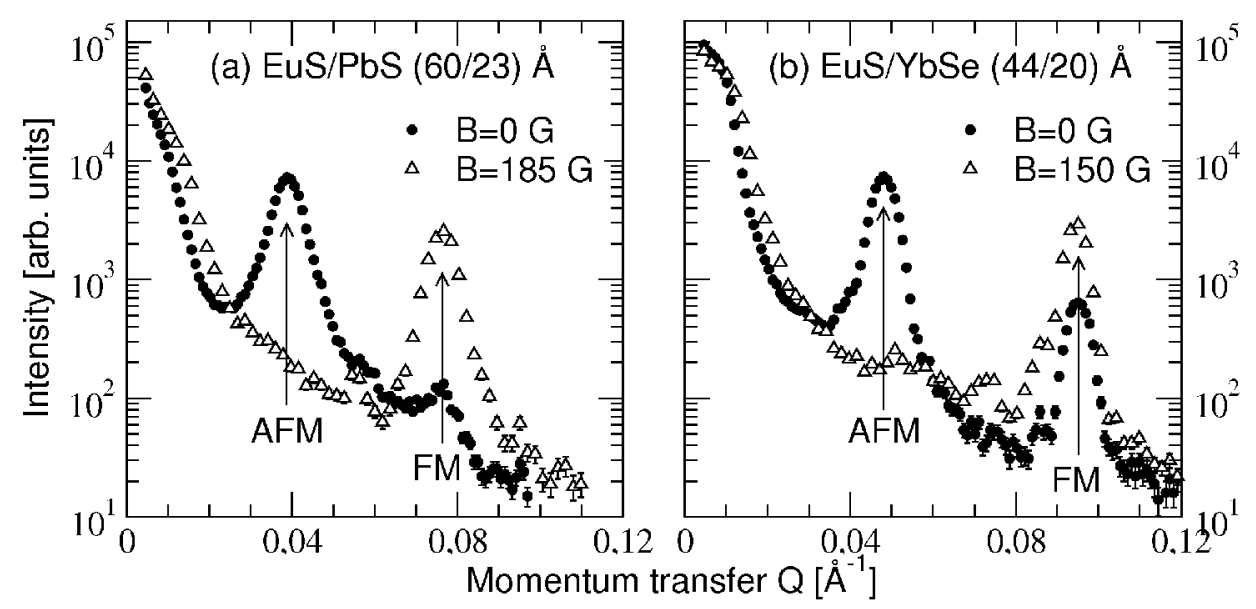

Fig. 1. Neutron reflectivity spectra for EuS/PbS (60/23) $\AA$ (a) and EuS/YbSe (44/20) $\AA$ (b) SL, measured below $T_{\mathrm{C}}(4 \mathrm{~K})$ in zero external field (circles) and in the saturating magnetic field of $185 \mathrm{G}$ or $150 \mathrm{G}$ (triangles).

The strength of the IC can be obtained from the value of the saturating magnetic field only if the coupling is much stronger than the in-plane anisotropy forces. In such case, the decrease in the AFM peak intensity for increasing field is fully reversible, when the external magnetic field is decreased back to zero. When the IC is comparable or weaker than the anisotropy forces, a hysteresis-like behavior should be observed. A complete restoration of an AFM interlayer configuration upon reversal of the direction of the applied magnetic field was observed for $\mathrm{EuS} / \mathrm{PbS}$ with very thin spacer layers. For EuS/PbS with a thicker $\mathrm{PbS}$ spacer, the AF M EuS interlayer configuration is restored only partially - due to the wider spacer layer, the IC is weaker and eventually becomes comparable to the anisotropy forces. In the case of EuS/YbSe SL with comparable YbSe spacer thicknesses the restored AFM peak intensities after the field reversal are considerably lower than for EuS/PbS SLs (compare Fig. 2 and Fig. 3). This observation is in qualitative agreement with the theoretical results obtained in Ref. [5]. To determine the experimental value of the IC constant, which can be compared with the theoretical model, the knowledge of the anisotropy fields in the studied structures is needed. 
(a) EuS/PbS (30/4.5) $\AA$

(b) EuS/PbS (35/12) ^

(c) EuS/PbS (45/25) $\AA$
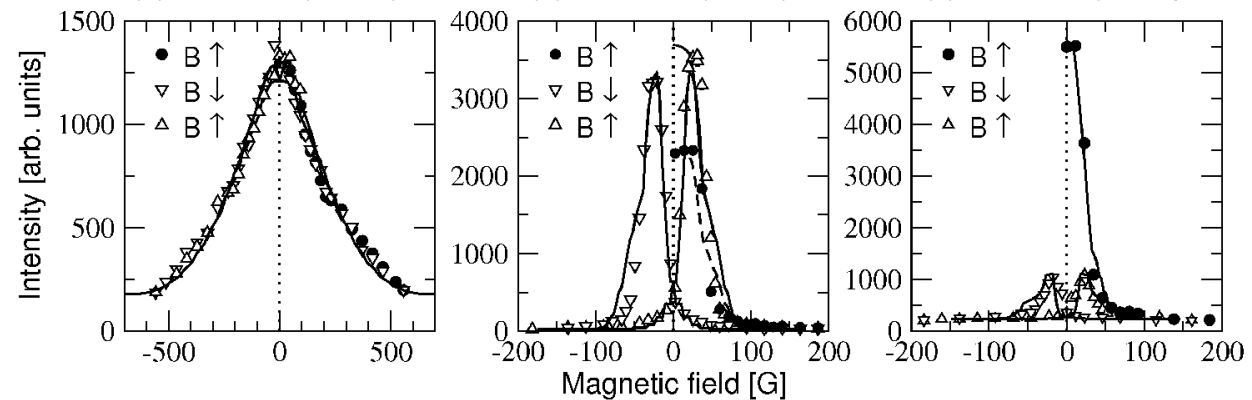

Fig. 2. The intensity of the AFM neutron reflectivity peak vs. magnetic field for three different $(\mathrm{EuS})_{m} /(\mathrm{PbS})_{n} \mathrm{SL}$ : (a) $m=10, n=1.5$; (b) $m=11.67, n=4$; (c) $m=$ $15, n=8.33$. The lines represent the fitting.

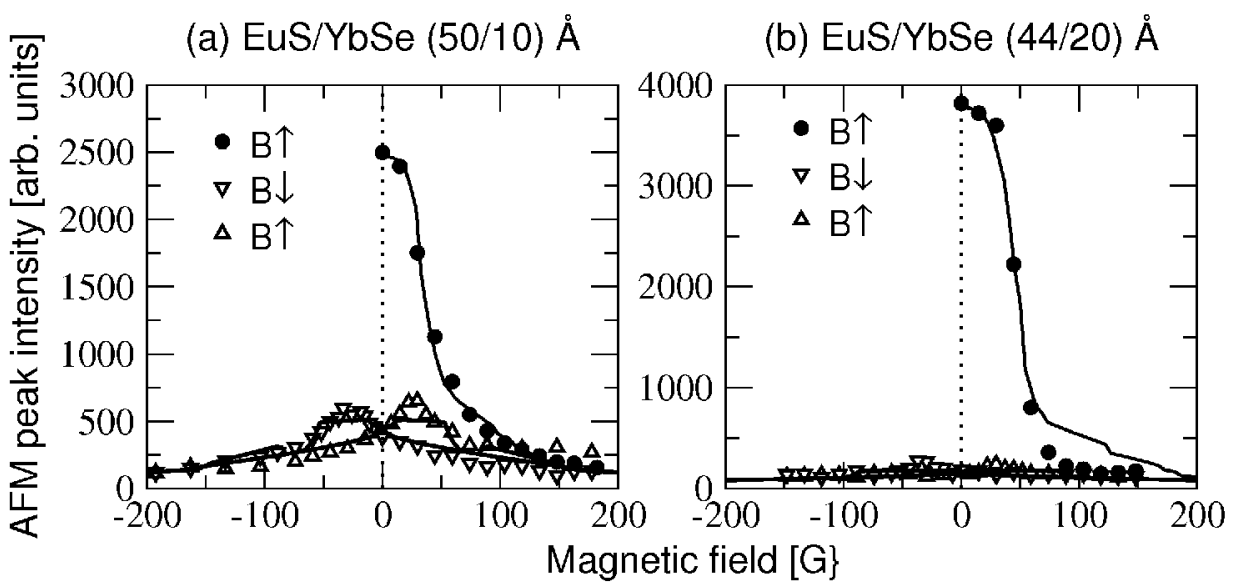

Fig. 3. The intensity of the AFM neutron reflectivity peak vs. magnetic field for two $(\mathrm{EuS})_{m} /(\mathrm{YbSe})_{n} \mathrm{SL}:$ (a) $m=16.67, n=3.33$; (b) $m=14.67, n=6.67$. The lines represent the fitting.

The in-plane domain structure and in-plane magnetic anisotropy in the EuS layers in EuS/PbS and EuS/YbSe SL were studied by specular polarized neutron reflectometry [6]. In bulk EuS the easy axes lie along [111]-type directions. In the layered structures, due to the shape anisotropy, the magnetization directions are confined to the (001) growth plane of the layers. The neutron reflectivity measurements, performed in conjunction with rotating the samples about the axis normal to the reflecting surface, essentially show the presence of the biaxial state with $90^{\circ}$ domains, in agreement with the fourfold in-plane symmetry of the EuS layer. Surprisingly enough, it was found, however, that the domain magnetizations in EuS/PbS and EuS/YbSe SLs were aligned along different in-plane directions, the easy axes being $\langle 210\rangle$ and $\langle 110\rangle$, respectively [6]. Moreover, in both kinds of 
SL the populations of the two types of domains are far from being equal - about three quarters of domains are oriented along one easy axis. This result suggests that the fourfold symmetry of the EuS layers in the studied SL is weakened as compared to the bulk crystal.

\section{Stoner-Wohlfarth model}

In order to describe the dependence of the intensity of the AFM neutron reflectivity peak vs. magnetic field in terms of IC and anisotropy the Stoner-Wohlfarth Hamiltonian [7] was used. We consider two FM layers, described by magnetization vectors $\boldsymbol{M}_{s, 1}$ and $\boldsymbol{M}_{s, 2}$. We assume that these magnetizations and the magnetic field lie in-plane, and thus the angles between magnetization vectors and magnetic field, $\theta_{1}$ and $\theta_{2}$, are sufficient to describe the system. The total magnetic energy of the layers is given by:

$$
E=E_{J}+E_{H, 1}+E_{H, 2}+E_{K, 1}+E_{K, 2} .
$$

It consists of IC described by the constant $J$ :

$$
E_{J}=-J \frac{\boldsymbol{M}_{s, 1} \cdot \boldsymbol{M}_{s, 2}}{M_{s, 1} M_{s, 2}}=-J \cos \left(\theta_{1}-\theta_{2}\right),
$$

the cubic anisotropy $K$ :

$$
E_{K, i}=-t K \cos ^{4} \theta_{i},
$$

and Zeeman terms:

$$
E_{H, i}=-t \mu_{0} \boldsymbol{M}_{s, i} \cdot \boldsymbol{H}=-t \mu_{0} M_{s} H \cos \left(\theta_{i}\right) .
$$

This energy was minimized as a function of the magnetization directions. The obtained directions were used to calculate the magnetic structure sums and to compute the intensity of AFM peaks. The values of the IC and the anisotropy constants, $J$ and $K$, were obtained by least-square fitting of the calculated peak intensities for different magnetic fields to the experimental data. The applied procedure is schematically shown in Fig. 4 . We note that for $J<K / 3$ the AFM configuration cannot be restored upon reversal of the magnetic field, as shown in Fig. 4a. On the other hand, when $J>K / 3$, the coupling is strong enough to restore the AFM alignment of the layers and leads to the spectrum presented in Fig. 4b. The observed spectra can be reproduced only by a combination of these two behaviors, as presented in Fig. 4c. Thus, in the fitting it was necessary to assume that different magnetic domains are coupled with different strengths (i.e., are separated by spacers of different thickness) - in other words, it was necessary to take into account the magnetic/nonmagnetic interfacial roughness. The population of the domains coupled by IC of different $J$ was described by a Gaussian distribution. We calculate the dependence of AFM peak intensity on magnetic field for a range of values of $J$ and sum up the results with appropriate weights. 

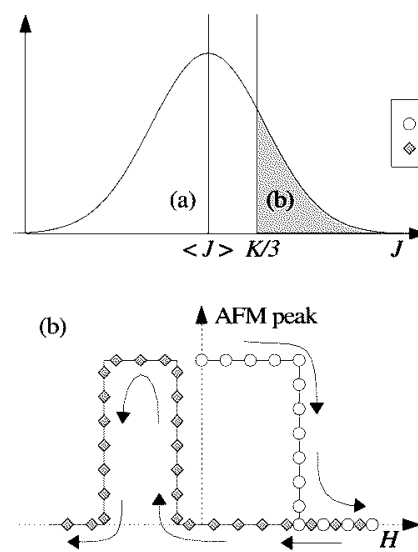

(a) AFM peak

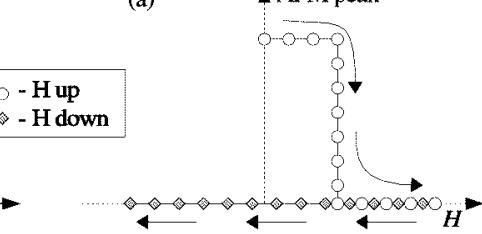

(c)

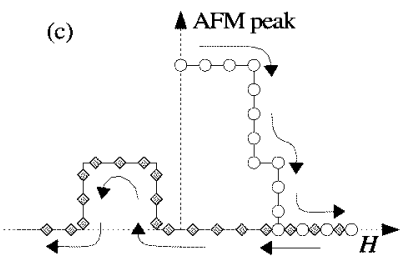

Fig. 4. The intensity of the AFM peak vs. magnetic field in perfect samples shows two types of behavior: (a) for small $J(J<K / 3)$ the coupling is too weak to restore AFM configuration when we reverse the magnetic field; (b) when $J>K / 3$ the coupling is able to restore AFM configuration. The observed AFM peak intensity dependence on magnetic field (c) is a combination of these two types of spectra with appropriate Gaussian weights.

In Figs. 2 and 3 the fitted spectra are presented by connected lines. For both EuS/PbS and EuS/YbSe SL, the best fit was obtained for the directions of the in-plane easy axes, which agree with those determined by polarized neutron reflectivity, i.e, $\langle 210\rangle$ and $\langle 110\rangle$, respectively. The addition of an uniaxial anisotropy term (along the directions determined by the polarized neutron experiments) to the Stoner-Wohlfarth Hamiltonian further improved the fitting for the EuS/YbSe structure.

The fitting procedure allowed us to extract from the neutron reflectivity results the strength of anisotropy forces in the SL. The obtained values of the anisotropy constant $K$ are similar for all samples of the same type of SL, but for $\mathrm{EuS} / \mathrm{YbSe} K$ is much bigger than for EuS/PbS. Moreover, it allowed one to determine the mean strength of IC, also for samples for which the AFM layer configuration was not fully restored upon reversal of the direction of the applied magnetic field. In Fig. 5, these values are presented (a) with those calculated within the theoretical model (b), for a variety of EuS/PbS and EuS/YbSe SL (mind the different scales in part (a) and (b)). As the inaccuracy of determining the $J$ constant by the fitting procedure depends crucially on the $J / K$ ratio, the results for SL with thicker spacers, i.e., smaller $J$, and especially for EuS/YbSe, are considerably overestimated. Moreover, the fitted values of $J$ for larger spacer thicknesses contain the contribution from the slowly decreasing dipolar coupling $[8,1]$. Thus, although the presented fitting enables the explanation of the neutron reflectivity experiments performed in the external magnetic field, the obtained by 


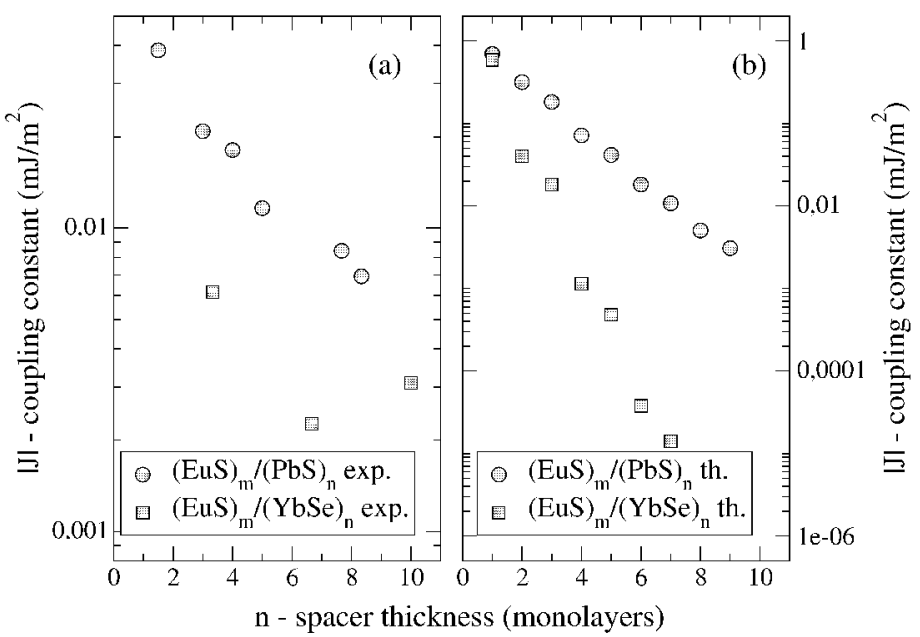

Fig. 5. The fitted (a) and model (b) strength of IC as a function of the spacer thickness for EuS/PbS (circles) and EuS/YbSe (squares) SL.

this procedure $J$ values cannot be compared constructively with the theoretical model $[4,5]$.

\section{Conclusions}

Using the Stoner-Wohlfarth Hamiltonian we have reproduced the dependence of the intensity of AFM neutron reflectivity peak vs. magnetic field, for a variety of EuS/PbS and EuS/YbSe SL. The necessity of including the spread of $J$ into the fitting procedure, finally proves the impact of the interfacial roughness on the strength of the interlayer correlations in real EuS-based SL structures. This may explain why the observed IC is usually an order of magnitude weaker than the theoretically predicted coupling for a perfect SL. It was first suggested in [4] that this discrepancy can be ascribed either to the strains resulting from the lattice mismatch between the SL constituent materials and the $\mathrm{KCl}$ substrate or, more likely, to the interfacial roughness. Further, the theoretical study of the dependence of IC on strain [5] seemed to exclude the first possibility. It was also shown in [3] that ICs in EuS/PbS/EuS trilayers, grown on either $\mathrm{PbS}$ or $\mathrm{KCl}$ substrates, do not differ significantly. The present study shows the need of including the SL imperfections in the theoretical description of the IC. The fitting also shows that the experiments performed in external magnetic field confirm the result obtained by polarized neutron reflectivity that in-plane easy axes in EuS/PbS and EuS/YbSe lie along different, $\langle 210\rangle$ and $\langle 110\rangle$, respectively, crystallographic directions. Finally, it was obtained that the spin-orbit interactions in $\mathrm{PbS}$ do not affect noticeably the theoretical values of the IC mediated by valence electrons. 


\section{Acknowledgments}

The support of FENIKS project (EC:G5RD-CT-2001-00535) and PBZ-KBN -044/P03/2001, NSF DMR-0204105 and CRDF (UP2-2444-KH-02) grants is gratefully acknowledged.

\section{References}

[1] H. Kępa, J. Kutner-Pielaszek, J. Blinowski, A. Twardowski, C.F. Majkrzak, T. Story, P. Kacman, R.R. Gałazka, K. Ha, H.J.M. Swagten, W.J.M. de Jonge, A.Yu. Sipatov, T.M. Giebultowicz, Europhys. Lett. 56, 54 (2001).

[2] H. Kępa, P. Sankowski, P. Kacman, C.F. Majkrzak, A.Yu. Sipatov, T.M. Giebultowicz, J. Magn. Magn. Mater. 323, 272 (2004).

[3] C.J.P. Smits, A.T. Filip, H.J.M. Swagten, B. Koopmans, W.J.M. de Jonge, M. Chernyshova, L. Kowalczyk, K. Grasza, A. Szczerbakow, T. Story, W. Palosz, A.Yu. Sipatov, Phys. Rev. B (2004) (in print).

[4] J. Blinowski, P. Kacman, Phys. Rev. B 64, 045302 (2001).

[5] P. Sankowski, P. Kacman, Acta Phys. Pol. A 103, 621 (2003).

[6] H. Kępa, C.F. Majkrzak, A.Yu. Sipatov, T.M. Giebultowicz, Physica B 345, 193 (2004)

[7] E.C. Stoner, E.P. Wohlfarth, Nature 160, 650 (1947).

[8] J.A. Borchers, P.M. Gehring, R.W. Erwin, J.F. Ankner, C.F. Majkrzak, T.L. Hylton, K.R. Coffey, M.A. Parker, J.K. Howard, Phys. Rev. B 54, 9870 (1996). 